\title{
History by numbers
}

God Created the Integers: The

Mathematical Breakthroughs that

Changed History

edited by Stephen Hawking

Running Press: $2005.1,160$ pp. $\$ 29.95$

\section{Jeremy Gray}

The subtitle - the mathematical breakthroughs that changed history - is not mere advertising. It is a yardstick, in fact various yardsticks, for assessing the book as a whole. Stephen Hawking brings us generous selections of Euclid's Elements, the works of Archimedes and Diophantus, René Descartes' Geometry entire, Pierre-Simon Laplace's $A$ Philosophical Essay on Probability, and material from Joseph Fourier, Carl Friedrich Gauss, Augustin-Louis Cauchy, Bernhard Riemann, Georg Cantor and Henri-Léon Lebesgue. There are also the first 11 chapters of George Boole's An Investigation into the Laws of Thought, Richard Dedekind's Essays on the Theory of Numbers, smaller extracts from Newton's Principia, and papers by Karl Weierstrass, Kurt Godel and Alan Turing. All these have some claim to have changed history, except perhaps the work by Diophantus, remarkable though it is. The same claim can be made of quite a few other works too, but as this book has nearly 1,200 pages it would be churlish to complain.

The items are well chosen. They are an interesting mix of the well known and the unexpected, and cover a range of topics in mathematics from geometry to mathematical analysis, probability and the modern foundations of mathematics. They range over an extensive period and, because many are presented either whole or at least extensively, they can be read with pleasure.

Every anthology faces the issue of what to do with the best-known pieces. Include them and some complain that they are too well known; omit them and others lament their loss. If this book is the only collection of original works of mathematics in translation that a reader owns, there is a lot to be said for it. But as an addition to the small but useful number of collections in English it is more annoying, because the opportunity was not taken to translate more works. All but four of the items here are already available in
English. The new ones are the work by Cauchy (some of which already exists in English elsewhere), some of the papers by Riemann (one of which is already in English), the passage by Weierstrass, and the extracts from the work of Lebesgue.

It is one thing to use an old translation if there is no significant improvement to be had in making a new one, but it is quite another to perpetuate an inadequate work. Here the Diophantus is annoying because it is the version by Thomas Little Heath. He turned the work into a densely written but by now old-fashioned school problem book, and the commentary, given in extensive footnotes, moves the reader even further from Diophantus' habit of mind. It is true that Diophantus' example is so strange that it reminds us how little we know about the ancient Greeks, but we do know how it was received in the Arab world and in the modern West, and it can be said to be only one of several works that helped bring about the 'modern algebra' of the seventeenth century. It would have been better if Hawking had included instead al-Khwarizmi's work on $a l$-jabr, from which algebra takes

\section{A culture of knowledge}

An exhibition in Paris explores the golden age of Islamic science.

Pete Jeffs

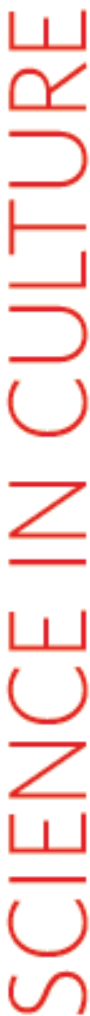

There is a mosque near my studio in east Paris. Passing by at the hour of prayer, I find myself intrigued by an Islamic culture that dates from the eighth century, occupies a central place in world culture, and yet remains a mystery for other societies.

The exhibition 'The Golden Age of Arabic Sciences', which can be seen at the Institute of the Arab World in Paris until 19 March, sheds lighton a people who produced exquisitemanuscripts, developed experimental sciences, and added new disciplines to those of the classical world.

The exhibition questions the idea that Arab-Islamic science simply translated key classical sources of knowledge and passed them on to the West. It shows how the Arabs of the expanding empire transformed and extended Greek, Mesopotamian, Persian and Indian ideas, and turned theory into practice. Analysis preceded assimilation.

The optics of lbnal-Haytham (AD 965-1040), for example, encouraged an experimental approach to the pursuit of physics. In 830 , al-Khwarizmi, the acknowledged founder of algebra, detailed the necessary mathematics for calculating inheritances, conducting commerce and constructing canals. Baconians would surely have approved. By this time, paper was becoming the preferred vector for the transmission of Arabic science. Industrial-scale production began around 750 , first in Samarkand and then in Baghdad. Cheap paper rapidly outstripped the use of papyrus and parchment. Almost 4 million individual Arabic writings from the eighth century onwards are known to have been conserved by libraries throughout the world.

Arab society integrated its knowledge by building hospitals, observatories and libraries, beginning with the House of Wisdom - Bayt a-Hikma - in Baghdad, shortly after 800 .

Translations and original writings were stored in more than a thousand different public and semi-public libraries scattered throughout the empire, catering for a widespread demand for scholarship. Knowledge was transmitted by scholars who travelled regularly, as well as through written correspondence. The Koran, incidentally, encouraged scientific activity. Some 200 manuscripts and objects have been assembled in Paris for the exhibition, including this planispheric astrolabe from the Nasser D. Khalili Collection and a profusely illustrated copy of Dioscorides' Herbal. But the exhibition opens with a map. When we can associate cities such as Damascus or Maragheh with astronomy as readily as we associate Padua with anatomy or Leiden with electricity, Arab-Islamic culture will be on its way to a fuller appreciation in the West. This exhibition offers a fine lesson in the meaning of the word 'civilization'.

Pete Jeffs is an artistbased in Paris, France. 
its name (see 'A culture of knowledge').

As for Eudid and Archimedes, there are 240 pages of Heath's editions with their erudite but dated commentaries. These may well help the reader with the mathematics, but historical scholarship has moved on. Unfortunately, the new comments are not much better. Each item is introduced with remarks that vary from the personal and insightful to the tired and incorrect. Among the latter is the supposed Greek crisis of the incommensurables, which was once presumed to have derailed the pythagoreans and which most historians these days think had little effect. The generally more accurate account of the life and work of Archimedes fails to mention the fact that the only manuscript of The Method - the most interesting of his works, in which he explained how he came to his discoveries - has recently re-entered the public domain after disappearing for most of the twentieth century.

Similar comments could be made about the more modern entries. These too are generally accurate but the origin of the information is not stated, so readers have no chance to catch up with contemporary scholarship in the history of mathematics. Nor can they find out how to sustain the flame of interest this book surely hopes to kindle, which is a pity, because Hawking's comments have an infectious enthusiasm for their subject and the book contains some great works.

Jeremy Gray is at the Centre for the History of the Mathematical Sciences, Faculty of Mathematics and Computing, OpenUniversity, Milton Keynes MK7 6AA, UK.

\section{Sex and power}

\section{Sexual Conflict \\ by Göran Arnqvist \& Locke Rowe \\ Princeton University Press: 2005. 360 pp. \\ $\$ 99.50, \in 55$ (hbk); \$39.50, £26.95 (pbk)}

\section{Tracey Chapman}

Sexual conflict occurs because of the different evolutionary interests of males and females in reproductive decisions. Indeed, the differential investment of males and females in reproductive episodes and the low relatedness of mating partners make sexual conflict almost inevitable. Geoff Parker clearly laid out the theoretical basis for sexual conflict in the 1970 s, but it is only over the past ten or so years that a significant body of empirical work has started to emerge.

There has, however, been little agreement about what sexual conflict is, or what might constitute unambiguous evidence for it. In addition, the relative importance of sexual conflict in driving evolutionary change, and the extent to which it could contribute to reproductive isolation and speciation, are unknown. There is also considerable confusion about precisely how coevolution driven by sexual conflict is distinct from traditional models of coevolutionary change by sexual selection (that which arises from competition between individuals of the same sex for matings, or from mate choice).

Into this uncertainty comes the excellent and wide-ranging Sexual Conflict by Göran Arnqvist and Locke Rowe, the first booklength treatment of this emerging field. The authors have done a great service in defining the field and in illuminating some of its conceptual difficulties. They also bring together a rich treasury of examples that must surely stimulate discussion and further study.

The greatest strength of the book is in tackling the theory. Arnqvist and Rowe do a first-rate job of dissecting models of sexual selection and sexual conflict, and in getting to grips with the distinctions between them. The book is well worth reading for this alone. For example, the reader is led clearly through the idea that coevolution driven by sexual conflict is distinct from sexual selection: under sexual

IMAGE
UNAVAILABLE
FOR COPYRIGHT
REASONS

Sexual partners often seem to be pulling in different directions.
Arnqvist and Rowe expose the core problem in the empirical study of sexual conflict: it is difficult to observe. It is common to see what looks like overt aggression or fights between male and female mating partners. But such observations by themselves do not necessarily indicate that sexual conflict is responsible and cannot, without further information, exclude the possibility of sexual selection. Arnqvist and Rowe highlight the fact

conflict, the selection on females is direct and focused on reducing female mating costs.

Such costs cannot be incorporated into the traditional models of sexual selection developed by R. A. Fisher in the 1930s, which propose a genetic benefit of female mate choice through the enhanced mating ability of sons. In contrast, costs of choice are not a problem for 'good genes' (indicator) models, but these assume that female choice will result in the acquisition of genetic fitness benefits for the offspring, and this is not generally assumed to occur under sexual conflict. The non-adaptive result of female mating biases also distinguishes sexual conflict from direct-benefit models.

Sexual conflict could therefore operate within this traditional sexual-selection framework but be distinguished from it by the nature of the forces acting on female mating biases. However, the authors also suggest that these standard equilibrium models may be inappropriate for analysing sexual conflict. For example, if males had a vast array of ways in which to manipulate females, and females had a similarly large number of ways in which to respond, the dynamics of this contest could well be more suited to non-equilibrium modelling. If this were true, then evolutionary change driven by sexual conflict would be different from any processes of mate choice with which we are familiar. that a process of continual adaptation and counter-adaptation could obscure evolutionary change resulting from sexual conflict. It may also be difficult to measure the costs and benefits of reproductive decisions under an appropriate range of relevant conditions, or even to decide what data are required. As the authors state, "unambiguous experimental data of sexual conflict are really quite scarce".

The best part of the ensuing discussion of the empirical studies in which sexual conflict could be operating covers the species for which good data are available, notably bed bugs, diving beetles, water-striders, fruitflies and dunnocks. The authors also consider numerous further cases in which sexual conflict might be occurring. These are more anecdotal but provide some fascinating natural history, such as the use of gin traps by male sage bush crickets, the 'playing dead'strategy of some species of robber fly, and the male funnel-web spider, which drugs its mate before copulation. This remarkable wealth of examples hints at the potentially ubiquitous distribution and importance of sexual conflict, and represents an extremely valuable resource that should stimulate further study and experimentation on the systems described. Tracey Chapman is in the Department of Biology, University College London, Gower Street, London WC1E6BT, UK. 